\title{
Relación Transversal, Vertical y Sagital en Sujetos con Deformidad Facial Candidatos a Cirugía Ortognática
}

\author{
Transverse, Vertical and Sagittal Relationship in Subjects \\ with Facial Deformity Candidates for Orthognathic Surgery
}

Víctor Ravelo'; Márcio de Moraes² \& Sergio Olate ${ }^{1,3}$

RAVELO, V.; DE MORAES, M.; OLATE, S. Relación transversal, vertical y sagital en sujetos con deformidad facial candidatos a cirugía ortognática. Int. J. Odontostomat., 14(4):664-669, 2020.

RESUMEN: El objetivo de este estudio fue evaluar la relación entre la clase esqueletal sagital y las condiciones transversales o verticales en sujetos con deformidad facial sin presencia de asimetría facial; Se realizó un análisis cefalométrico de la clase esqueletal sagital, transversal y vertical en 115 sujetos con indicaciones de cirugía ortognática. Se consideró algunos datos del análisis cefalométrico de Steiner y el análisis cefalométrico de Ricketts en sentido sagital para determinar la clase esqueletal facial. A nivel transversal se determinó la dimensión transversal facial, dimensión transversal maxilar y dimensión transversal mandibular. A nivel vertical se determinó la dimensión vertical oclusal y dimensión vertical total. Al comparar las mediciones transversales y verticales entre sujetos de sexo femenino y masculino, se observó que los hombres presentaban mayores dimensiones que las mujeres $(p=0,0001)$ en todos los análisis realizados. Los sujetos clase III presentaron mayor dimensión transversal facial $(p=0,0002)$ y transversal mandibular $(p=0,001)$ que los sujetos clase II. Además, se observó que los sujetos clase III presentaban mayor dimensión vertical total $(p=0,002)$ que los sujetos clase II; Es posible concluir que existe características faciales transversales y verticales que se pueden relacionar con la posición sagital de las estructuras maxilo-mandibulares.

PALABRAS CLAVE: deformidad facial, cirugía ortognática, asimetría facial, cefalometría.

\section{INTRODUCCIÓN}

La deformidad facial genera un desequilibrio importante en diferentes componentes del macizo facial, y puede verse enmascarada por compensación dentaria, de tejidos blandos y cambios posturales (Baek et al., 2012).

La apariencia facial tiene un conjunto de componentes esqueléticos y blandos, que al encontrarse alteradas pueden generar alteraciones sagitales, verticales y transversales que en conjunto son alteraciones complejas de la forma facial. Las asimetrías se pueden ver expresadas con mayor prevalencia en el tercio medio implicando alteraciones en el maxilar que impactan en la morfología nasal (Yi \& Jang, 2015), como también en el tercio inferior de la cara, siendo esta última el que presenta mayor prevalencia debido a que es un hueso móvil que crece por un mayor pe- riodo de tiempo con dos polos intensos de crecimiento (cóndilos mandibulares) en comparación a la maxila (Nur et al., 2016).

Las condiciones a nivel sagital, transversal y/o vertical influyen drásticamente en el biotipo facial. En sujetos que presentan discrepancias vertical y/o transversal, se observa hipo o hiperdivergencia mandibular y un rostro más ancho o alargado (Nanda, 1990; Ha et al., 2014). Esta morfología se acompaña de manifestaciones en los tejidos blandos como alteraciones en las estructuras labiales, sobre erupciones e inclinaciones dentarias excesivas (Murakami et al., 2016).

Debido a que estas alteraciones tienen un componente esqueletal, es necesario realizar tratamientos quirúrgicos para devolver la función y los

\footnotetext{
${ }^{1}$ Centro de Excelencia en Estudios Morfológicos y Quirúrgicos (CEMyQ), Universidad de La Frontera, Chile.

2 División de Cirugía Oral y Maxilofacial, Facultad de Odontología de Piracicaba, Universidad Estadual de Campinas, Chile.

${ }^{3}$ División de Cirugía Oral, Facial y Maxilofacial, Facultad de Odontología, Universidad de La Frontera, Chile.
} 
estándares de normalidad de proporción estética, los cuales pueden variar dependiendo de la moda, cultura y la etnia (Kim et al., 2015). Al realizar cirugía ortognática es importante identificar los cambios sagitales, transversales y verticales involucrados en la deformidad para poder obtener resultados eficientes en la devolución de la estética facial (Yáñez-Vico et al., 2011; Kogou et al., 2018).

El objetivo de este estudio fue evaluar la relación entre la clase esqueletal sagital y las condiciones transversales o verticales en sujetos con deformidad facial sin presencia de asimetría facial.

\section{MATERIAL Y MÉTODO}

Se realizó un análisis de la deformidad facial de 115 sujetos obteniendo comparaciones entre la clase esqueletal sagital y su relación transversal y vertical previo al inicio de ortodoncia preparatorio para cirugía ortognática. Los sujetos incorporados firmaron un consentimiento informado y accedieron voluntariamente a participar en el estudio. La investigación se realizó resguardando la integridad de los participantes y respetando la Declaración de Helsinki.

Fueron incluidos sujetos de ambos sexos que presentaran deformidad esquelética clase II y clase
III. Se excluyeron sujetos con cirugía facial previa, antecedentes de trauma máxilo facial, presencia de síndromes o condiciones faciales de significativa alteración morfológica y sujetos con asimetrías faciales definidas por la desviación del mentón de más de 5 $\mathrm{mm}$ desde la línea mediana facial.

Se realizó el análisis cefalométrico de Steiner en sentido sagital para determinar la clase esqueletal facial y el análisis cefalométrico de Ricketts en sentido postero-anterior; a nivel transversal se determinó la dimensión transversal facial (DTF), dimensión transversal maxilar (DTMx) y dimensión transversal mandibular (DTMn) (Fig. 1). A nivel vertical se determinó la dimensión vertical oclusal (DVO) y dimensión vertical total (DVT) (Fig. 2).

Análisis Sagital y Clase Esqueletal. Se utilizo el análisis de Steiner para determinar la angulación A-Na-B (Punto A: ubicado en la zona más profunda de la concavidad anterior del hueso maxilar; Nasion: ubicado en la intersección de las suturas internasal y frontal; Punto B: ubicado en el punto más profundo de la concavidad anterior del maxilar). El parámetro de normalidad fue un ángulo de $2^{\circ}$; angulación mayor a $2^{\circ}$ como una clase esqueletal II y una angulación menor a $2^{\circ}$ como una clase esqueletal III.

\section{Análisis Transversal Coronal. Dimensión transver-} sal facial: Se consideró la distancia entre los puntos
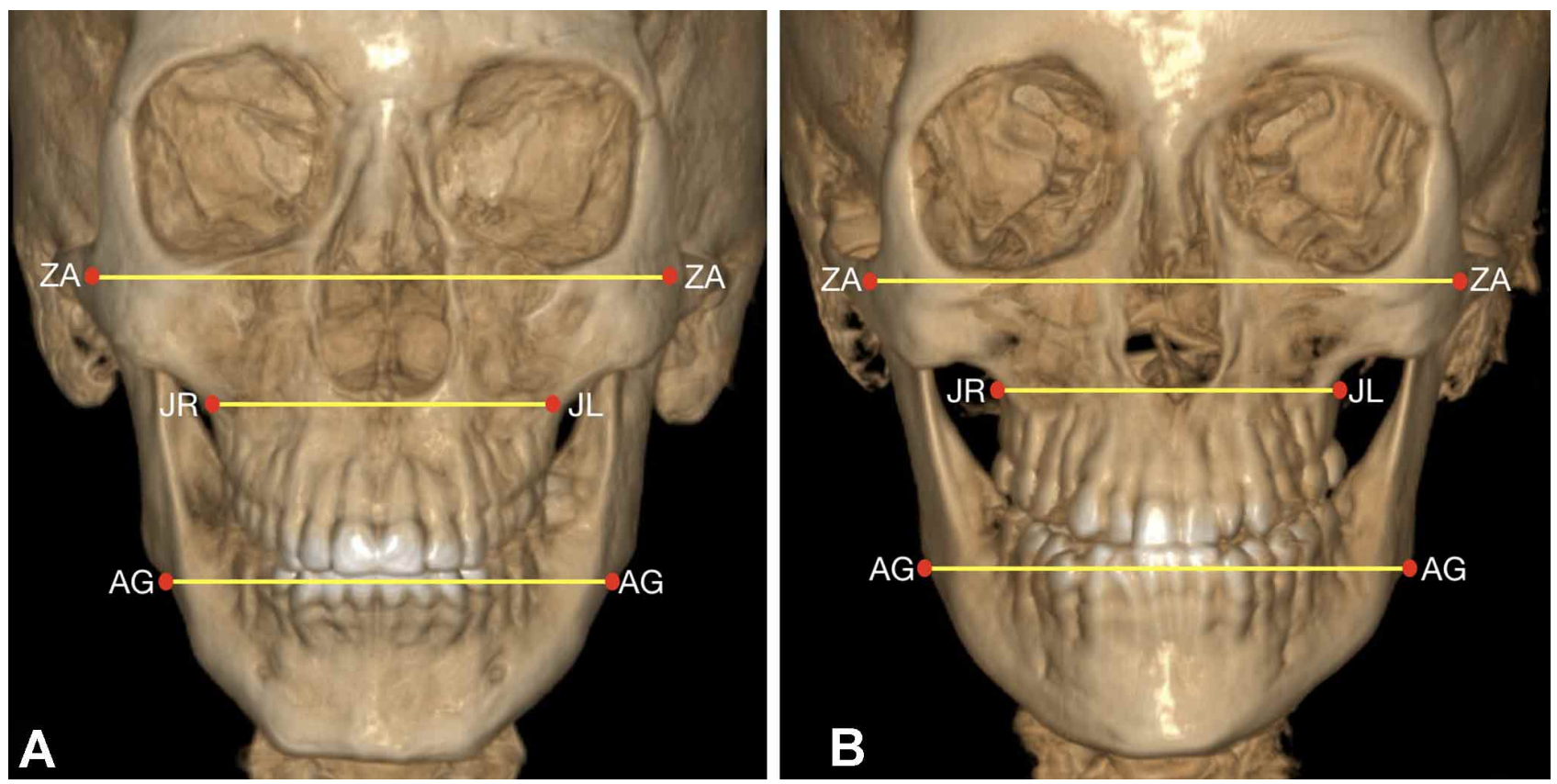

Fig. 1. Morfometría facial realizada en sujetos clase II (A) y clase III (B). Dimensión transversal facial (DTF), dimensión transversal maxilar (DTMx) y dimensión transversal mandibular (DTMn). 

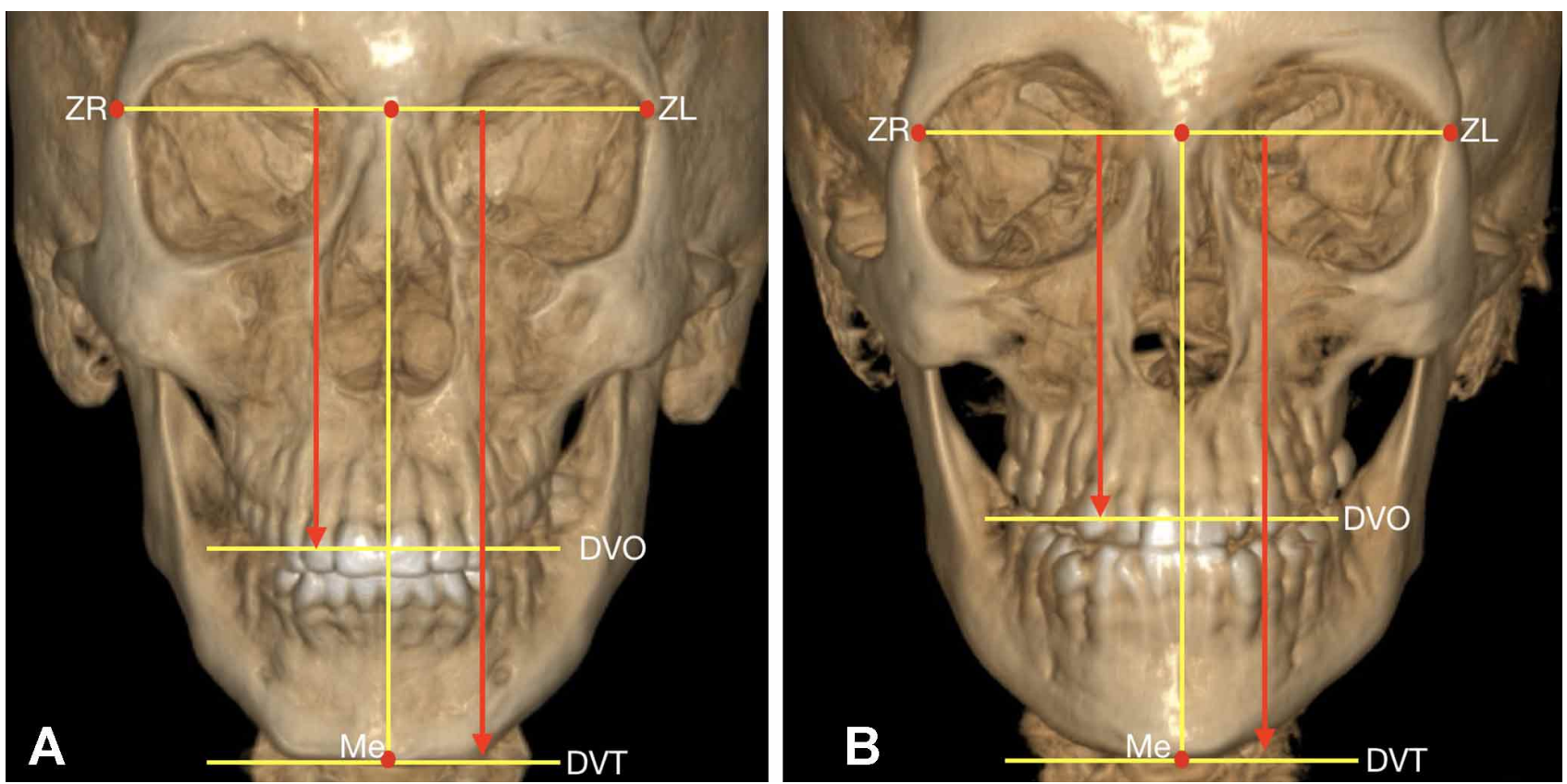

Fig. 2. Morfometría facial realizada en sujetos clase II (A) y clase III (B) para determinar la dimensión vertical oclusal (DVO) y dimensión vertical total (DVT).

Za-Za para determinar la distancia transversal facial (punto más lateral y superior del arco cigomático).

Dimensión transversal maxilar: Distancia que expresa el crecimiento transversal del maxilar. Se consideró la distancia entre los puntos JR-JL (intersección del punto más inferior del hueso cigomático con la tuberosidad del maxilar).

Dimensión transversal mandibular: Distancia que expresa el crecimiento transversal mandibular. Se consideró la distancia entre los puntos AG-AG (puntos que se localizan entre los contornos gonial y antegonial en el margen inferior de la rama mandibular).

\section{Sentido Vertical Coronal}

Dimensión vertical oclusal: Se consideró el punto mediano entre los puntos ZR-ZL (línea transversal que une las suturas frontocigomáticas derecha e izquierda), desde donde se trazó la perpendicular hasta el punto mediano de la línea transversal formada por el plano oclusal molar.

Dimensión vertical total: Se consideró el punto mediano entre los puntos ZR-ZL (línea transversal que une las suturas frontocigomáticas derecha e izquierda), desde donde se trazó la perpendicular hasta el punto Me (Mentón: margen inferior de la sínfisis, localizada inferiormente a la protuberancia mental).
Los resultados fueron tabulados y sometidos a análisis estadístico. Inicialmente se realizó un estudio de análisis intraclase para comparar las mediciones obteniendo un resultado de 0,9 . Posteriormente, se utilizó un intervalo de confianza del $95 \%$ para medir la concordancia entre las mediciones transversales, verticales y la deformidad esqueletal facial. Además, se realizo el Test Spearman para determinar la correlación entre variables. Se consideró un valor de $p<0,05$ para determinar la diferencia significativa.

\section{RESULTADOS}

Los sujetos incluidos presentaron una edad de $27,8 \pm 11,6$ años (rango de 18 a 63 años). 46 (40\%) sujetos eran de sexo masculino mientras que $69(60 \%)$ eran de sexo femenino. Según la clasificación de Steiner, 71 sujetos $(61,7 \%)$ fueron clase II y 44 sujetos $(38,3 \%)$ fueron clase III.

Al analizar las mediciones transversales y verticales entre sujetos de sexo femenino y masculino (Tabla I), se observó que los sujetos de sexo masculino presentaron mayores dimensiones que los sujetos de sexo femenino $(p=0,0001)$ en todos los análisis realizados.

Cuando se comparó la clase esqueletal y las mediciones transversales, se observó que los sujetos cla- 
se III presentaron mayor dimensión transversal facial $(p=0,0002)$ y dimensión transversal mandibular $(p=0,001)$ que los sujetos clase II (Tabla II). Pero al analizar la dimensión transversal maxilar, esta no se correlacionó con la clase esqueletal $(p=0,21)$ y tampoco hubo diferencia en el promedio de las mediciones en los sujetos clase II y clase III.
Además, al comparar la clase esqueletal y las mediciones verticales, los sujetos clase III presentaron mayor dimensión vertical total $(p=0,002)$ y dimensión vertical oclusal que los sujetos clase II (Tabla III). Pero, esta última medición no presentó diferencia estadística con la clase esqueletal $(p=0,14)$.

Tabla I. Distribución de datos entre el sexo de los individuos y el promedio de mediciones transversales y verticales.

\begin{tabular}{lccccc}
\hline & \multicolumn{5}{c}{ Sexo } \\
\cline { 2 - 6 } & \multicolumn{3}{c}{ Masculino } & Femenino \\
\hline MEDICIONES & X & DS & X & DS & P $<0.05$ \\
\hline Dimensión transversal facial & $83,86 \mathrm{~mm}$ & 3,96 & $79,66 \mathrm{~mm}$ & 3,87 & $0.0001^{*}$ \\
Dimensión transversal maxilar & $54,86 \mathrm{~mm}$ & 3,07 & $53,02 \mathrm{~mm}$ & 2,98 & $0.0001^{*}$ \\
Dimensión transversal mandibular & $84,86 \mathrm{~mm}$ & 5,43 & $79,66 \mathrm{~mm}$ & 5,57 & $0.0001^{*}$ \\
Dimensión vertical oclusal & $66,89 \mathrm{~mm}$ & 4,60 & $63,68 \mathrm{~mm}$ & 3,96 & $0.0001^{*}$ \\
Dimensión vertical total & $105,89 \mathrm{~mm}$ & 8,37 & $100,65 \mathrm{~mm}$ & 5,35 & $0.0001^{*}$ \\
\hline
\end{tabular}

* diferencia estadísticamente significativa.

Tabla II. Distribución de datos entre la clase esqueletal y mediciones transversales.

\begin{tabular}{|c|c|c|c|c|c|}
\hline \multirow[b]{3}{*}{ MEDICIONES TRANSVERSALES } & \multicolumn{4}{|c|}{ Clase esqueletal } & \multirow[b]{3}{*}{$P<0.05$} \\
\hline & \multicolumn{2}{|c|}{ Cll } & \multicolumn{2}{|c|}{ CIII } & \\
\hline & $\mathrm{X}$ & DS & $X$ & DS & \\
\hline Dimensión transversal facial & $80,21 \mathrm{~mm}$ & 4,15 & $83,18 \mathrm{~mm}$ & 4,22 & $0.0002^{*}$ \\
\hline Dimensión transversal maxilar & $54,28 \mathrm{~mm}$ & 3,29 & $54,54 \mathrm{~mm}$ & 2,72 & 0.21 \\
\hline Dimensión transversal mandibular & $80,40 \mathrm{~mm}$ & 6,33 & $83,90 \mathrm{~mm}$ & 4,83 & $0.001^{*}$ \\
\hline
\end{tabular}

CII. Retrognático; CIII. Prognático; * diferencia estadísticamente significativa.

Tabla III. Distribución de datos entre la clase esqueletal y mediciones verticales.

\begin{tabular}{lccccc}
\hline & \multicolumn{5}{c}{ Clase esqueletal } \\
\cline { 2 - 6 } & \multicolumn{2}{c}{ CII } & \multicolumn{7}{c}{ CIII } & DS & $\mathrm{P}<0.05$ \\
\hline MEDICIONES VERTICALES & $\mathrm{X}$ & DS & $\mathrm{X}$ & 0.14 \\
\hline Dimensión vertical oclusal & $64,60 \mathrm{~mm}$ & 4,74 & $65,64 \mathrm{~mm}$ & 4,07 & $0.002^{*}$ \\
Dimensión vertical total & $101,22 \mathrm{~mm}$ & 7,06 & $105,18 \mathrm{~mm}$ & 6,72 & 0.72
\end{tabular}

CII. Retrognático; CIII. Prognático; * diferencia estadísticamente significativa.

\section{DISCUSIÓN}

El análisis cefalométrico en dos dimensiones (2D) ha sido utilizado por mucho tiempo para realizar mediciones lineales y angulares de tejidos duros y blandos del complejo cráneo facial, pero con la incorporación de la tomografía computarizada de haz cónico (CBCT), se han planteado diferentes posibilidades de planificar, tratar y realizar seguimientos de forma eficiente (Porto et al., 2014), debido a que las mediciones tridimensionales generan menor distorsión por asimetría que las imágenes en 2D (Gateño et al., 2011).
En nuestro estudio realizado con CBCT, se observó la correlación que existe entre los sujetos con deformidad facial clase II y clase III y las mediciones transversal facial, transversal mandibular y medición vertical facial total. A pesar de la no inclusión de sujetos con deformidad facial asimétrica, se observó que los sujetos clase III presentaron mayor asimetría que los sujetos clase II, probablemente relacionado con su mayor diámetro esqueletal. 
Nuestros resultados permiten señalar que en sujetos clase III existe un mayor ancho facial, mayor ancho mandibular pero no un mayor ancho maxilar cuando comparamos con sujetos de clase II esqueletal, lo que indica que el aumento transversal de la maxila no se relaciona con la clase esqueletal del sujeto; de la misma forma, sujetos con clase III esqueletal presentaron un rostro más alto a expensas probablemente del tamaño mandibular ya que la altura vertical de la maxila no se relacionó con la clase esqueletal. Esto indica que la anomalía esqueletal de clase III evaluada en el sentido coronal, existe un crecimiento general mayor del rostro con excepción del crecimiento de la maxila, mientras que en sujetos clase II existe una proporción vertical más equilibrada entre los diferentes componentes.

Nuestros datos concuerdan con varios autores (Bayome et al., 2013; Thiesen et al., 2017a; Leung \& Leung, 2018) quienes observaron una correlación entre la medición transversal de la cara con la longitud vertical facial. La asimetría facial en sujetos con clase III esqueletal era mayor que en los sujetos clase II, siendo el componente mandibular el que presentar mayor implicancia en el biotipo facial. Kwon et al. (2006) realizaron un análisis de 42 sujetos con y sin asimetría facial, en donde observaron una correlación positiva entre la posición sagital maxilo-mandibular en los sujetos con asimetría facial, y una mayor prevalencia de desviación mandibular con un promedio de $10,7 \mathrm{~mm}$ en comparación con los sujetos sin asimetría facial.

Allareddy et al. (2016) mencionaron que pueden existir múltiples variaciones en las manifestaciones maxilo mandibulares pero que el plano oclusal y ángulo mandibular son factores importantes ya que pueden relacionarse con mordidas abiertas o invertida, y que estos factores pueden aumentar en el tiempo. Nuestro estudio no se encontró correlación entre la clase esqueletal facial y el plano vertical oclusal debido a que nuestra investigación realiza un análisis en puntos maxilares laterales únicos y no verdaderamente en un plano oclusal sagital como Allareddy et al. Por otra parte, la asimetría facial clase III es evidenciada debido a la proyección del mentón y el ángulo mandibular prominente, lo que da una apreciación más ancha de la cara, pero en el caso de los pacientes clase II, puede existir un encubrimiento de sus características esqueletal debido a la compensación de los tejidos blandos y variaciones étnicas (Thiesen et al., 2017b; Rana et al., 2017).

El objetivo de la cirugía ortognática es corregir las deformidades maxilo mandibulares para lograr una correcta relación funcional maxilo mandibular, relación oclusal y proporción facial adecuada, pero este aspecto facial se ven fuertemente influenciado por la limitada predictibilidad de los tejidos blandos (Lo et al., 2018), en donde la respuesta de estos tejidos posterior a la cirugía y a la cantidad de cambios necesarios no son totalmente predecibles en el sector medio de la cara, mientras que en el tercio inferior y región mandibular presenta una correlación mayor y mayor predictibilidad que en el maxilar (Wermker et al., 2014). Aunque existan métodos que predicen cambios de los tejidos blandos, estos no son confiables debido a la versatilidad de estos tejidos, por lo que aun no deben usarse como presentación final para predecir resultados faciales (Olate et al., 2017).

Choi et al. (2014) utilizando un escáner facial 3D en 25 pacientes con deformidad facial y operados con cirugía ortognática, determinaron que el ancho cigomático no presentaba cambios posteriores a la cirugía, pero al evaluar la altura facial vertical y el ancho bigonial existía una diferencia significativa, determinando que la rotación anteroposterior mandibular es la que disminuye el ancho bigonial y la altura facial.

Sun et al. (2018) mencionaron que los pacientes con deformidades faciales presentan una autoestima más baja que la población normal, además de una capacidad funcional disminuida, condición que mejora significativamente posterior a la cirugía facial. En este sentido es importante realizar estas intervenciones para mejorar la calidad de vida de los pacientes ya que mejora la percepción física, relaciones sociales, condición y función laboral y cuadros depresivos tanto en pacientes adolescentes como adultos (Gabardo et al., 2019).

Es posible concluir que existen características faciales transversales y verticales que se pueden relacionar con la posición sagital de las estructuras maxilomandibulares. Sujetos clase III presentan mayor tamaño facial transversal y vertical con excepción de la maxila, que no se diferencia con la clase sagital tipo II o tipo III.

RAVELO, V.; DE MORAES, M.; OLATE, S. Transverse, vertical and sagittal relationship in subjects with facial deformity candidates for orthognathic surgery. Int. J. Odontostomat., 14(4):664-669, 2020.

ABSTRACT: The aim of this study is to evaluate the relationship between sagittal skeletal class and transverse or vertical conditions in subjects with facial deformity without presence of facial asymmetry; A comparative study was conducted between the results of the cephalometric analysis of the sagittal, transverse and vertical skeletal class in 115 
subjects with indications for orthognathic surgery. The Steiner cephalometric analysis in the sagittal direction was used to determine the facial skeletal class and the Ricketts cephalometric analysis in the sagittal analysis. At the transverse level it was determined the facial transverse dimension, maxillary transverse dimension and mandibular transverse dimension. At a vertical level it is determined the occlusal vertical dimension and total vertical dimension. When comparing cross-sectional and vertical measurements between female and male subjects, it was observed that male subjects presented greater dimensions than female subjects $(p=0.0001)$. Class III subjects present greater facial transverse dimension $(p=0.0002)$ and mandibular transverse dimension $(p=0.001)$ than class II subjects. Also, it was observed that class III subjects had a greater total vertical dimension $(p=0.002)$ than class II subjects; It is possible to conclude that there are facial characteristics transverse and vertical that can be related with the sagittal position of the maxillo-mandibular structures.

KEY WORDS: facial deformity, orthognathic surgery, facial asymmetry, cephalometry

\section{REFERENCIAS BIBLIOGRÁFICAS}

Allareddy, V.; Ching, N.; Macklin, E. A.; Voelz, L.; Wintraub, G.; Davidson, E.; Prock, L. A.; Rosen, D.; Brunn, R. \& Skotko, B. G. Craniofacial features as assessed by lateral cephalometric measurements in children with Down syndrome. Prog. Orthod., 17:35, 2016

Baek, C.; Paeng, J. Y.; Lee, J. S. \& Hong, J. Morphologic evaluation and classification of facial asymmetry using 3-dimensional computed tomography. J. Oral Maxillofac. Surg., 70(5):1161-9, 2012.

Bayome, M.; Park, J. H. \& Kook, Y. A. New three-dimensional cephalometric analyses among adults with a skeletal Class I pattern and normal occlusion. Korean J. Orthod., 43(2):62-73, 2013.

Choi, J. W.; Lee, J. Y.; Oh, T. S.; Kwon, S. M.; Yang, S. J. \& Koh, K. S. Frontal soft tissue analysis using a 3 dimensional camera following two-jaw rotational orthognathic surgery in skeletal class III patients. J. Craniomaxillofac. Surg., 42(3):220-6, 2014.

Gabardo, M.; Zielak, J.; Tórtora, G.; Gerber, J.; Meger, M.; Rebellato, N.; Küchler, E. \& Scariot, R. Impact of orthognathic surgery on quality of life: Predisposing clinical and genetic factors. J. Craniomaxillofac. Surg., 47(8):1285-91, 2019.

Gateño, J.; Xia, J. J. \& Teichgraeber, J. F. Effect of facial asymmetry on two-dimensional and three-dimensional cephalometric measurements. J. Oral Maxillofac. Surg., 69(3):655-62, 2011.

Ha, Y.; Park, Y. S. \& Lee, S. P. Do long-faced subjects really have a long anterior face? A longitudinal study. Am. J. Orthod. Dentofacial Orthop., 145(6):799-806, 2014.

Kim, S. Y.; Bayome, M.; Park, J. H.; Kook, Y. A.; Kang, J. H.; Kim, K. H. \& Moon, H. B. Evaluation of the facial dimensions of young adult women with a preferred facial appearance. Korean J. Orthod., 45(5):253-60, 2015

Kogou, T.; Takaki, T. \& Shibahara, T. Three-dimensional analysis and evaluation in orthognathic surgical cases with facial asymmetry. Bull. Tokyo Dent. Coll., 59(3):147-61, 2018.

Kwon, T. G.; Park, H. S.; Ryoo, H. M. \& Lee, S. H. A comparison of craniofacial morphology in patients with and without facial asymmetry--a three-dimensional analysis with computed tomography. Int. J. Oral Maxillofac. Surg., 35(1):43-8, 2006.

Leung, M. Y. \& Leung, Y. Y. Three-dimensional evaluation of mandibular asymmetry: a new classification and three-dimensional cephalometric analysis. Int. J. Oral Maxillofac. Surg., 47(8):104351, 2018.

Lo, L. J.; Weng, J. L.; Ho, C. T. \& Lin, H. H. Three-dimensional regionbased study on the relationship between soft and hard tissue changes after orthognathic surgery in patients with prognathism. PLoS One, 13(8):e0200589, 2018.

Murakami, T.; Kataoka, T.; Tagawa, J.; Yamashiro, T. \& Kamioka, H. Antero-posterior and vertical facial type variations influence the aesthetic preference of the antero-posterior lip positions. Eur. J. Orthod., 38(4):414-21, 2016.

Nanda, S. K. Growth patterns in subjects with long and short faces. Am. J. Orthod. Dentofacial Orthop., 98(3):247-58, 1990.

Nur, R. B.; Çakan, D. G. \& Arun, T. Evaluation of facial hard and soft tissue asymmetry using cone-beam computed tomography. Am. J. Orthod. Dentofacial Orthop., 149(2):225-37, 2016.

Olate, S.; Zaror, C. \& Mommaerts, M. Y. A systematic review of softto-hard tissue ratios in orthognathic surgery. Part IV: 3D analysis Is there evidence? J. Craniomaxillofac. Surg., 45(8):1278-86, 2017.

Porto, O. C. L.; de Freitas, J. C.; Gonçalves de Alencar, A. H. \& Estrela, C. The use of three-dimensional cephalometric references in dentoskeletal symmetry diagnosis. Dental Press J. Orthod., 19(6):78-85, 2014.

Rana, N.; Qu, Y. Y.; Yao, W. \& Liu, L. Comparison of cephalometric hard and soft tissues of adolescents with angle class II division 1 malocclusion between Northern Chinese population and Northern Indian population. Chin. J. Dent. Res., 20(1):33-42, 2017.

Sun, H.; Shang, H. T.; He, L. S.; Ding, M. C.; Su, Z. P. \& Shi, Y. L. Assessing the quality of life in patients with dentofacial deformities before and after orthognathic surgery. J. Oral Maxillofac. Surg., 76(10):2192-201, 2018.

Thiesen, G.; Gribel, B. F.; Freitas, M. P. M.; Oliver, D. R. \& Kim, K. B. Craniofacial features affecting mandibular asymmetries in skeletal Class II patients. J. Orofac. Orthop., 78(5):437-45. 2017b.

Thiesen, G.; Gribel, B. F.; Kim, K. B. \& Freitas, M. M. M. Maxillofacial features related to mandibular asymmetries in skeletal class III patients. J. Oral Maxillofac. Surg., 75(5):1015-25, 2017a.

Wermker, K.; Kleinheinz, J.; Jung, S. \& Dirksen, D. Soft tissue response and facial symmetry after orthognathic surgery. J. Craniomaxillofac. Surg., 42(6):e339-45, 2014.

Yáñez-Vico, R. M.; Iglesias-Linares, A.; Torres-Lagares, D.; GutiérrezPérez, J. L. \& Solano-Reina, E. Three-dimensional evaluation of craniofacial asymmetry: an analysis using computed tomography. Clin. Oral Investig., 15(5):729-36, 2011.

Yi, J. S. \& Jang, Y. J. Frequency and characteristics of facial asymmetry in patients with deviated noses. JAMA Facial Plast. Surg., 17(4):265-9, 2015.

Corresponding author:

Prof. Sergio Olate, DDS, PhD

Claro Solar 115, Oficina 414-A

Division of Oral and Maxillofacial Surgery

Universidad de La Frontera

Temuco

CHILE

Email: sergio.olate@ufrontera.cl

Received:06-06-2020

Accepted:08-07-2020 